\title{
Antiherpetic treatment and the improvement of pemphigus lesions
}

\section{Hajjar Daakir, Line Mezni, Karima Senouci, Nadia Ismaïli, Meriam Meziane, Laila Benzekri}

\author{
Department of Dermatology and Venerology IBN SINA hospital, Mohamed V Rabat University, Morocco
}

Corresponding author: Hajjar Daakir, MD, E-mail: Hajjar20daakir@gmail.com

Sir,

Pemphigus is a group of autoimmune blistering diseases of the skin and/or mucous membranes caused by the presence of antibodies against adhesion molecules on the cell surface of keratinocytes [1]. In genetically predisposed patients, several factors, including drugs, physical agents, neoplasms, hormones, and viruses, notably herpes simplex virus (HSV).

Through this study, we expose the impact of Herpes Simplex Virus infection on our patients with pemphigus

This prospective study, which lasted around 15 months (between September 2019 and November 2020). We collected 8 medical records of hospitalized patients with pemphigus associated with HSV infection admitted to the dermatology unit of Ibn Sina University Hospital of Rabat Morocco.

To exploit the data collected, an operating sheet was prepared to include; identity, age, sex, pathological history, history of the disease, and characteristics of herpetic infection evolution of pemphigus and herpetic infection.

In our 8 cases of pemphigus associated with HSV infection, the age of the patients varied between 31 and 67 years with a median of 48 years.

The sex ratio was 1 . The clinical forms of pemphigus are distributed as follows: One Seborrheic, three Foliaceous, four Vulgar (50\% of cases) with a PDAI score that was between 23 and 102 .

The duration of pemphigus progression ranged from 30 days to 2 years with a median of 5.5 months
(2 patients were hospitalized for relapse and the 6 others were newly diagnosed).

In all our patients Pemphigus was confirmed on skin biopsy, Direct immunofluorescence, and the research of antibodies anti-intercellular substance via IFI which was positive in 7 of our patients $(87.5 \%)$ with a value between 320 and 1280, one was negative.

The treatment of pemphigus in our patients was based on general corticosteroids at a daily dose ranging from 1.5 to $2 \mathrm{mg} / \mathrm{kg}$ per day depending on the severity of pemphigus. This corticosteroid therapy was associated with immunosuppressive therapy in seven patients: azathioprine in four cases and rituximab in three cases.

HSV infection occurred 6 days before corticosteroids treatment in 1 case and 7 cases after the onset of general corticosteroids; the date of occurrence varied between 6 days and 84 days with a median of 27.5 days after the start of general corticosteroids at a dose of 1.5 to $2 \mathrm{mg} / \mathrm{kg}$ per day.

Clinically the HSV infection had the appearance of bullous vesicles in 2 patients, pruritic crusts in 4 patients, and mucous cutaneous erosions in 2 patients.

In all patients, the Tzanck smear was positive objectifying a cytopathogenic effect of type herpes virus,

2 of our patients received aciclovir intravenously at $10 \mathrm{mg} / \mathrm{kg} / 8 \mathrm{~h}$ for 8 to 12 days and 6 patients received oral valaciclovir at 1 to $3 \mathrm{~g} /$ day for 7 to 10 days.

Recovery was rapid for all patients treated with acyclovir or valaciclovir with the drying of herpes lesions and 


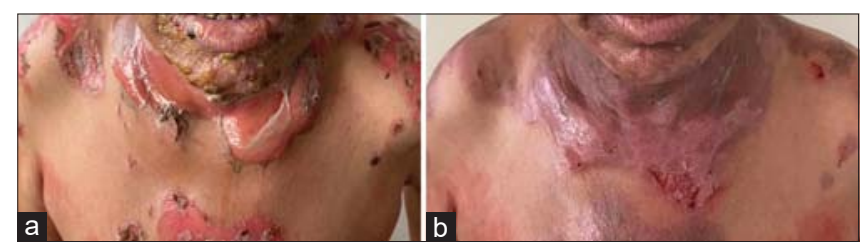

Figure 1: ( $a$ and $b)$ Pemphigus lesions with HSV infection, (b) Impovement of pemphigus lesions after antoherpetic treatement.

improvement of pemphigus lesions after one week of treatment (Fig. la and lb).

The possible association between herpesviruses and PV has been the subject of several studies; however, it remains unclear what's the role that the herpesviruses play in the progression and presentation of PV. Some studies were unable to identify associations between viral infections and pemphigus resulting from immunosuppressive therapies and/or a causative factor associated with treatmentresistant lesions. Oppositely, other studies suggested that herpesviruses could trigger PV progression [2]. The most common viral infections reported in pemphigus patients are caused by the herpesviruses, particularly HSV.

The mechanism of viral induction of autoimmunity can be explained in several ways, including molecular mimicry (cross-reactions between pathogen and self-derived epitopes), which seems the acceptable explanation for the association of viral infections and pemphigus. Further explanation, the viruses upregulate the production of interferon and interleukins. The high levels of interferon-gamma lead to an increased expression of HLA2 on keratinocytes, making the pemphigus antigen structural site active. Also, overproduction of IL4 and IL1 0 causes a shift of THl to TH2 response which potentiates antibody response [3].

On the other hand, Pemphigus disease is caused by autoantibodies directed against desmosomal cadherins desmoglein 1 (Dsgl) and desmoglein 3 (Dsg3) [1] and some studies prove that the defects in epidermis intercellular adhesion especially the E-cadherin facilitates access of HSV-l to its receptors to penetrate and initiate infection [4].

In this descriptive study, the presence of fissures, erosions, and hemorrhagic crusts are significant clinical markers of HSV infection among pemphigus patients. Antiherpetic treatment leads to the improvement of pemphigus lesions even in severe pemphigus.

Clinically, it might be difficult or even impossible to identify herpetic infections in PV patients. These observations suggested the clinical diagnostic of herpetic infection helps to avoid unnecessary changes of immunosuppressive treatment for presumed refractory pemphigus and to start an effective antiviral treatment without delay. It would be interesting for future studies to investigate the presence of HSV on skin specimens of patients affected by pemphigus and not receiving any immunosuppressive therapy. In addition, serological testing is required to search for the presence of specific serum IgM and IgG antibodies directed against the HSV in newly diagnosed pemphigus patients to disclose the possible occurrence of a primary HSV infection, as well as to further evaluate the potential role of this virus in inducing pemphigus. We also suggest looking for infections caused by other herpesviruses such as CMV, VZV, EBV, or HHV-8, HHV-6, and HHV7 in resistant lesions. Even though the clinical relationship between the presence of these viruses and PV is difficult to establish, an increase of the immunosuppressive doses could be detrimental to the patients $[4,5]$.

\section{Statement of Human and Animal Rights}

All the procedures followed were in accordance with the ethical standards of the responsible committee on human experimentation (institutional and national) and with the 2008 revision of the Declaration of Helsinki of 1975.

\section{Statement of Informed Consent}

Informed consent for participation in this study was obtained from all patients.

\section{REFERENCES}

1. Saito M, Stahley SN, Caughman CY, Mao X, Tucker DK, Payne AS, et al. Signaling dependent and independent mechanisms in pemphigus vulgaris blister formation. PlosOne. 2012;7:e50696.

2. Senger P, Sinha AA. Exploring the link between herpes viruses and pemphigus vulgaris: literature review and commentary. Eur J Dermatol. 2012;22:728-35.

3. Ruocco E, Ruocco V, Lo Schiavo A, Brunetti G, Wolf R. Viruses and pemphigus: an intriguing never-ending story. Dermatology. 2014;229:310-5.

4. Konda D, Chandrashekar L, Dhodapkar R, Ganesh RN, Thappa DM. Clinical markers of herpes simplex virus infection in patients with pemphigus vulgaris. J Am Acad Dermatol. 2019:S0190-9622:30905-3.

5. Rahn E, Thier K, Petermann P, Rübsam M, Staeheli P, Iden S, et al. Epithelial Barriers in Murine Skin during Herpes Simplex Virus 1 Infection: The role of tight junction formation. J Invest Dermatol. 2017;137:884-93.

Copyright by Hajjar Daakir, et al. This is an open-access article distributed under the terms of the Creative Commons Attribution License, which permits unrestricted use, distribution, and reproduction in any medium, provided the original author and source are credited.

Source of Support: Nil, Conflict of Interest: None declared. 